\title{
ARQUITECTURA Y PAISAJE SONORO DE UN ASENTAMIENTO INKA EN EL NOROESTE ARGENTINO
}

\author{
ARCHITECTURE AND SOUNDSCAPE OF AN INKA SETTLEMENT \\ IN NORTHWESTERN ARGENTINA
}

\author{
Alejandro Ferrari ${ }^{1}$, Iván Leibowicz², Joaquín Izaguirre ${ }^{3}$ y Félix Acuto ${ }^{2}$
}

\begin{abstract}
Este artículo presenta nuevos datos e interpretaciones respecto del modo en que los Inkas utilizaron la arquitectura para manipular las experiencias somáticas de las comunidades que habitaban el valle Calchaquí Norte (Salta, Argentina) a partir del control de su participación en espacios ritualizados. En esta línea, nos focalizaremos en las experiencias disímiles que estos espacios promovieron, ya sea a partir del estímulo de ciertas modalidades sensoriales sobre otras o mediante la secuenciación e intensidad con las que ellas intervinieron. Recientemente, y en respuesta al ocularcentrismo predominante en esta clase de investigaciones, se ha planteado la necesidad de integrar sentidos adicionales a la vista y avanzar hacia paisajes sonoros. Este trabajo expone la permeabilidad visual y las propiedades acústicas de la plaza de un pequeño asentamiento predominantemente Inka a partir de ensayos experimentales in situ y el modelado tridimensional de la arquitectura y de la dispersión de vectores de sonido. Propondremos que una cuidadosa planificación destinada a espacializar grupos y prácticas no se circunscribió a habilitar e inhibir selectivamente la accesibilidad visual a la plaza, sino que además utilizó la accesibilidad auditiva para crear diferentes experiencias entre sus visitantes.

Palabras claves: conquista Inka, espacialidad, arquitectura, paisajes sonoros.
\end{abstract}

This article presents new data and interpretations on how Inka representatives utilized the architecture of ritualized spaces to manipulate local communities' somatic experiences. In an attempt to overcome the prevailing ocularcentrism among this line of inquiry, it has been suggested that additional senses should be considered, and some scholars have begun to advance towards the study of ancient soundscapes. This article will focus on the plaza of an Inka settlement located in the North Calchaquí Valley (Salta, Argentina) paying specific attention to how it privileged certain sensorial modalities and managed their sequential stimulus and intensity. It article presents an analysis that combines on-field visual and acoustical permeability data generation, with three-dimensional models of architecture, terrain, and sound propagation. Results indicate that a careful layout design not only spatialized groups and practices, but also created different experiences by promoting disparate visual and acoustic stimulations upon approaching and entering the plaza.

Key words: Inka conquest, spatiality, architecture, soundscapes.

Numerosos estudios sobre contacto cultural y colonialismo han examinado el modo en que los colonizadores emplean materialidades y espacialidades para estructurar prácticas, desarticular $\mathrm{y}$ rearticular interacciones interpersonales, $\mathrm{y}$ promover la difusión e internalización de una cosmovisión hegemónica (Alcock et al. 2001; Cosgrove 2008; Morris y Scheidel 2009). Ciertos espacios y eventos en particular, como las ceremonias públicas, constituyen arenas esenciales para la transmisión de narrativas transformativas. En ellas, las multiplicidades se hacen manifiestas, las identidades se transforman y reafirman, y los sujetos incorporan un nuevo estrato a su biografía experiencial, adicional al compendio de acciones cotidianas que involucran a la vida ordinaria de una comunidad (Connerton 1989; Harvey 1994).

Por supuesto, las ceremonias públicas necesitan de un soporte material, espacial, y performativo a partir del cual articular su inteligibilidad, guiando y canalizando las experiencias de sus participantes. La arquitectura, uno de los principales correlatos materiales respecto de la temporalidad y espacialidad del habitar, puede y suele ser utilizada para cooptar el despliegue público, la performance, y el ritual, creando así auténticos teatros de poder que moldean el compendio de disposiciones que los sujetos adquieren (Inomata y Coben 2006; Pallasmaa 2005).

1 Consejo Nacional de Investigaciones Científicas y Técnicas-Instituto de Estudios Americanistas Julián Cáceres Freyre (Universidad Austral, Argentina). Cerrito 1250, C1010AAZ, Buenos Aires, Argentina. alejandroferra@gmail.com

2 Instituto Multidisciplinario de Historia y Ciencias Humanas-Consejo Nacional de Investigaciones Científicas y Técnicas. Saavedra 15, Piso 5, C1083ACA, Buenos Aires, Argentina. pinocarriaga@ hotmail.com; facuto@ gmail.com

3 Facultad de Filosofía y Letras, Universidad de Buenos Aires, Argentina. Puán 470. C1406CQJ. Buenos Aires, Argentina. joaquin.izaguirre@gmail.com 
Ya que lo cotidiano y lo eventual operan como escalas entrelazadas de entendimiento y actividad, la configuración de los espacios que se le revelan al sujeto en la totalidad de su habitar lo afectan en la dimensión física y psicológica y operan sobre los sentidos coordinando la producción, reproducción $\mathrm{y}$ transferencia de experiencias y emociones a partir del despliegue contextual de significaciones (Howes 2006; Soja 1989; Tarlow 2000). En este esquema, canalizar el movimiento y controlar la sensorialidad, resulta una herramienta fundamental para el control colectivo e individual de la experiencia, incidiendo sobre aquellas cosas (sensu Ingold 2010) con las que el sujeto entra en relación y sobre un devenir mnemónico que naturaliza distinciones y diagrama su reproducción (Bell 1992; Bourdieu 2007; Harvey 1994).

$\mathrm{Al}$ respecto, se ha señalado la necesidad de una arqueología de los sentidos que trate el modo en que las personas producían sus subjetividades e identidades colectivas a partir de las modalidades sensoriales incentivadas e inhibidas a lo largo de su vida. Una arqueología de los sentidos que logre vincular modalidades sensoriales privilegiadas y su cambio en el tiempo trasciende la dicotomía entre lo ritual y lo mundano, lo ordinario y lo extraordinario, e integra la cotidianeidad y la eventualidad en una pregunta relacional respecto del modo en que la cultura material se ve implicada en cambios experienciales (Hamilakis 2002, 2011). Uno de los principales desafíos para lograr este objetivo es lidiar con el ocularcentrismo prevalente en la arqueología y complementarlo con el estudio de otras modalidades sensoriales (Fahlander y Kjellström 2010).

Pese a que el concepto de paisaje sonoro entró en juego hacia fines de 1960 (Schafer 1967; Southworth 1969), solo en los últimos años ha sido crecientemente incorporado a la arqueología como potencial línea de evidencia (Karampatzakis y Zafranas 2009; McBride 2014; Reznikoff 2012; Watson y Keating 1999; entre otros). Por paisaje sonoro se entiende el agregado del total de la energía sonora en un contexto determinado con énfasis en el modo en que es percibido y entendido por aquellos que lo habitan (Truax 1984 [1978]), e incluye aquellas biofonías, antropofonías y geofonías que ocurren en un contexto dado y crean coocurrencias acústicas concretas (Pijanowski et al. 2011).

Superar el desafío de transcender el ocularcentrismo implica al menos y como punto de partida, evaluar el modo en que un contexto dado pudo haber estimulado sentidos adicionales a la vista. Luego, el modo en que la arquitectura pudo establecer ciertas correlaciones entre modalidades sensoriales, estimulando a las personas de modo secuencial, progresivo y, en intensidades diferentes.

En esta aproximación, evaluaremos las propiedades arquitectónicas, visuales y acústicas de un pequeño asentamiento predominantemente Inka emplazado en el valle Calchaquí Norte (Salta, Argentina. VCN de aquí en adelante). Nos focalizaremos en la accesibilidad a la plaza y la plataforma ceremonial emplazada en ella para entender si el diseño de este espacio, además de promover y censurar ciertas vistas para los oficiantes y los participantes de los eventos públicos, pudo haber aprovechado y regulado la propagación de sonido para incentivar experiencias distintas entre los asistentes.

\section{Plazas y Ceremonias Inkaicas: Políticas Imperiales de Difusión, Inclusión y Distinción}

Las políticas imperiales desplegadas a la hora de anexar diferentes grupos al Tawantinsuyu han sido uno de los tópicos más discutidos por aquellos investigadores que indagan en la expansión Inka, sus estrategias para comunicar la cosmología imperial, el correlato material en las regiones anexadas y, su éxito relativo. La incorporación de nuevos grupos a la esfera del Tawantinsuyu implicó complejas interacciones entre representantes imperiales y locales y, por lo tanto, gran variabilidad en las estrategias para negociar y legitimar el control político y económico mientras se articulaban necesidades y objetivos locales y foráneos (Bauer 1992; D'Altroy 2002; Murra 1978; Stanish 1992; entre muchos otros). Una estrategia inkaica recurrente fue incentivar y controlar la participación local en algunas de las ceremonias auspiciadas por los representantes imperiales. En particular, aquellas llevadas adelante en un escenario mayoritariamente asociado al despliegue de la ideología imperial y a la producción y reproducción de categorías sociales: las plazas.

Investigaciones arqueológicas, etnoarqueológicas y etnográficas han mostrado la importancia crucial de las plazas en la dinámica social andina en general e inkaica en particular (Makowski et al. 2005; Moore 1996; Nielsen 2006; entre muchos otros). Mediante las ceremonias que se llevaban 
adelante en ellas, se organizaba el trabajo de las comunidades, se validaban distinciones sociales, se actualizaban las relaciones con las entidades no humanas de la cosmología andina, y se difundía y contextualizaba la narrativa imperial. Las plazas Inka han sido definidas, en líneas generales, como grandes espacios abiertos delimitados por muros independientes y/o por las fachadas de las estructuras adyacentes. Independientemente de que se hayan documentado superficies que abarcan desde 19,2 ha (ver Morris et al. 2011 para Huánuco Pampa) hasta 0,1 ha (ver Leibowicz 2012 para La Huerta, quebrada de Humahuaca, Argentina), a menudo ocupan una posición central respecto del asentamiento y están relacionadas con arquitectura pública y de poder (Hyslop 1990; Morris y Covey 2003; Niles 1999). Más allá de su variabilidad formal, su importancia nuclear en la participación imperial y provincial y las actividades que allí se llevaban a cabo ha sido ampliamente documentada. Los cronistas que presenciaron y registraron algunas ceremonias nos legaron aspectos cruciales respecto del modo en que la participación estaba finamente coreografiada. La organización e identificación en función de la filiación étnica de los asistentes por medio de su vestimenta, adornos corporales, instrumentos, cantos y danzas tradicionales, era un aspecto obligado de las ceremonias en las plazas (Garcilaso de la Vega 1985:6:39-40 [1609]; Molina 2011:38 [fines del siglo XVI]).

$\mathrm{Al}$ interior de las plazas Inka la espacialización de personas y actividades estaba pautada o dirigida fundamentalmente por la posición de su plataforma ceremonial о иshnu (cuando presente). Al igual que las plazas, los ushnus presentan una gran variabilidad formal a lo largo del imperio y por ello se trata de uno de los componentes arquitectónicos más difíciles de estandarizar (ver p.ej. Hyslop 1990). En líneas generales, se trata de plataformas, cimas aplanadas o afloramientos naturales y rocas (con y sin modificaciones antrópicas) a las que se les han asignado variadas funcionalidades (tronos para la autoridad máxima, observaciones astronómicas, y la realización de ofrendas, libaciones y sacrificios), así como formas y extensiones (Farrington y Zapata 2003; Monteverde Sotil 2011; Pino Matos 2005; entre muchos otros). Pese a la variabilidad formal, ciertos aditamentos recurrentes como escalinatas, receptáculos para libar, y canaletas de drenaje, proveían las herramientas fundamentales para que el Inka o su representante vinculara y se comunicara con las entidades no humanas que habitaban los planos celeste (Hanan Pacha), terrenal (Kay Pacha), y del mundo de abajo (Ukhu Pacha) (Meddens et al. 2008; Staller 2008; Zuidema 1989). De modo que, así como las plazas resultaban el pivote fundamental de muchos asentamientos, los ushnus en su interior (cuando presentes) articulaban y significaban las actividades que se desarrollaban en ella. Al realizar ofrendas y conducir las actividades desde el ushnu, los Inkas insertaban a las poblaciones locales y así mismos en una narrativa más amplia que comunicaba la autoridad del Tawantinsuyu y su competencia a la hora de mediar entre las personas y las entidades no humanas, asegurando el bienestar de las primeras y apaciguando a las segundas.

\section{El Arribo Inka al Valle Calchaquí Norte: Guitián en Contexto Regional}

Los fechados disponibles indican que el noroeste argentino fue anexado al Tawantinsuyu hacia la primera mitad del siglo XV. En el valle Calchaquí Norte (Figura 1), si bien los representantes imperiales parecen haber evitado emplazar sus asentamientos más importantes en la cercanía del foco de ocupación local (para detalle ver Acuto 1999), hacia el sur del VCN emprendieron la construcción de Guitián (SSalCac 2) (Figura 2). Este asentamiento, cuya superficie intramuros abarca aproximadamente 1,6 ha, podría considerarse parte integral de un complejo mayor completado por otros dos asentamientos. Hacia el sur y hacia el oeste, en una terraza adyacente al río La Paya y a tan solo $50 \mathrm{~m}$ de los muros perimetrales de Guitián, se documentaron complejos de estructuras muy deteriorados cuyo diseño de planta y material de superficie nos permite adscribirlo preliminarmente al período Intermedio Tardío (1.000-1.430/1.450 d.C.). Hacia el suroeste, a tan solo 345 m, se ubica La Paya (SSalCac 1), un asentamiento de aproximadamente 6 ha que presenta mayoritariamente conjuntos de estructuras locales circunvalados por senderos elevados, y en la franja más elevada del sitio, un complejo de estructuras que aloja uno de los mejores ejemplos de arquitectura Inka en el noroeste argentino: Casa Morada (Ambrosetti 1907; González y Díaz 1992; entre otros). En este contexto regional se ha sugerido que la construcción de Guitián tuvo como propósito llevar adelante ceremonias cuyo objetivo fue reproducir la cosmología Inka a nivel local (Acuto 2010). También, que el sector Inka supone 


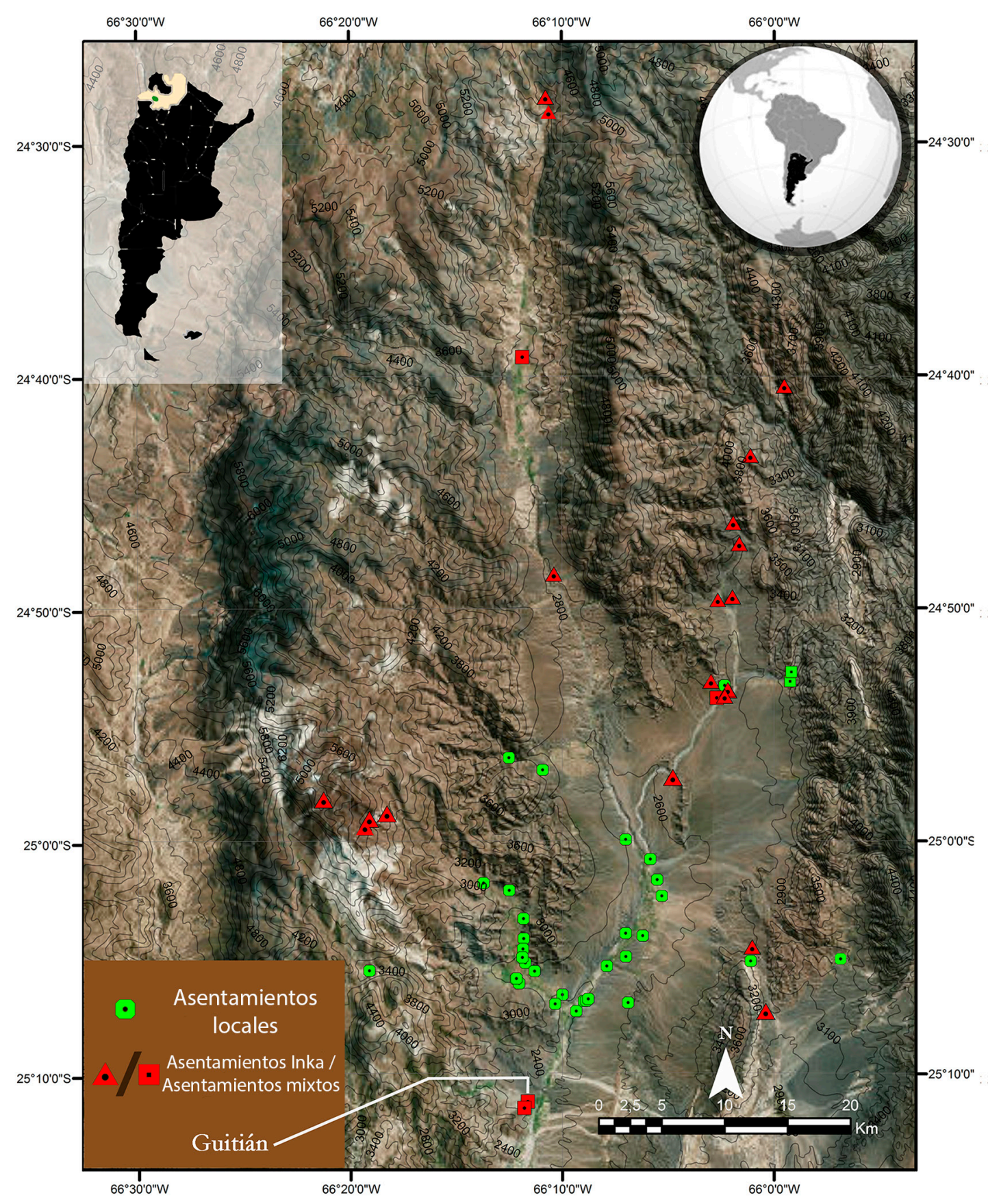

Figura 1. Área de estudio y localización del asentamiento analizado.

Area under study and location of analyzed settlement.

una intrusión en un asentamiento local previo en un área en que existían vínculos sociopolíticos más cercanos (Williams 2004).

Guitián consta de una zona Inka y una zona local. La primera de ellas se compone de una plaza central delimitada por complejos inkaicos (entre ellos, una kallanka en su lado oeste, dos kanchas en su lado norte) y un muro en su lado sur. La segunda zona, en la periferia oriental y septentrional de la superficie intra-muros, presenta conjuntos 


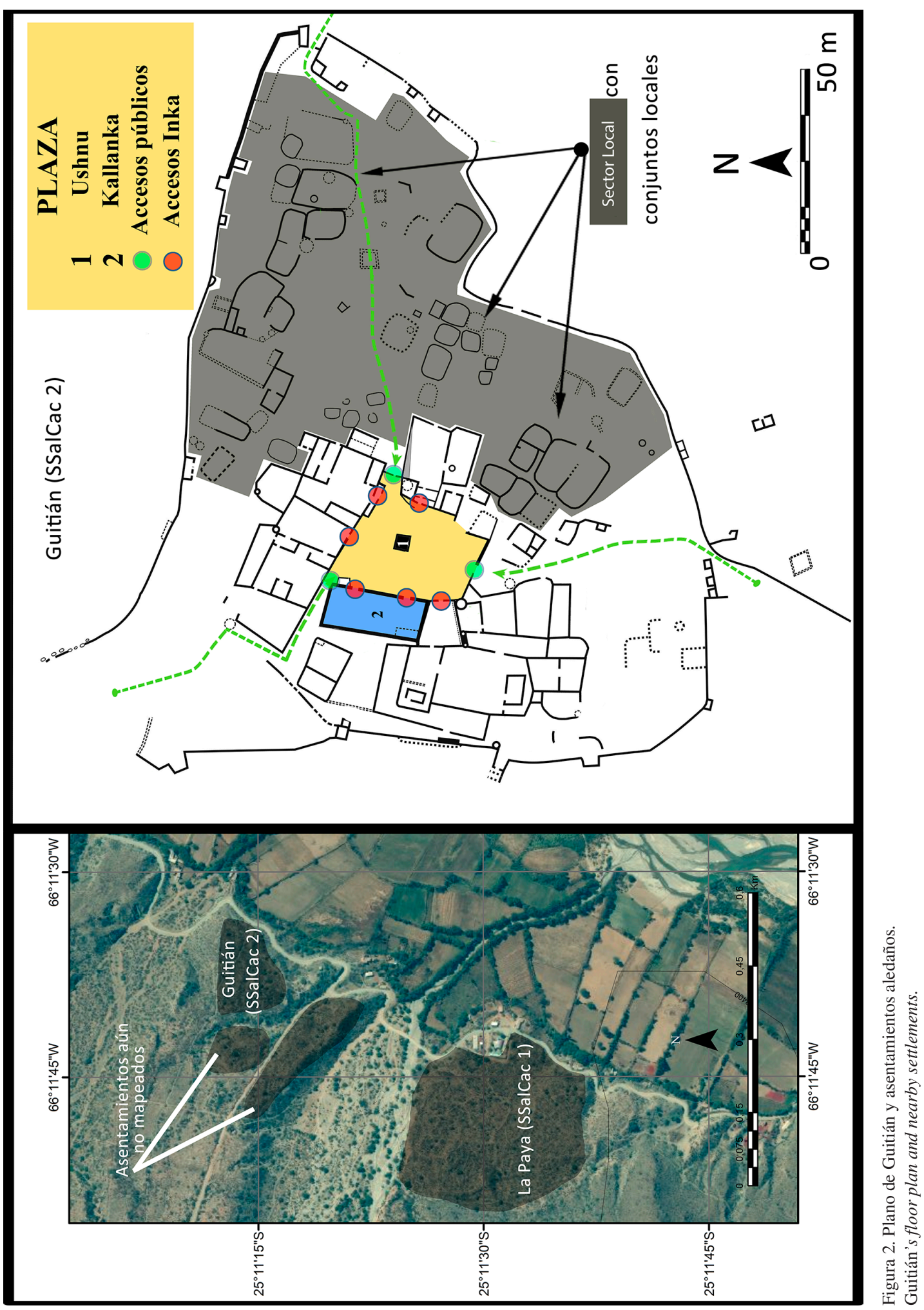


de estructuras cuyas características constructivas obedecen a las regularidades observadas para otros sitios locales de la región ${ }^{1}$.

Guitián se articula alrededor de una plaza central dentro de la que se emplazó, hacia el centro pero ligeramente desplazada hacia el norte, una plataforma ceremonial o ushnu que actualmente podemos estimar en $16 \mathrm{~m}^{2}$. En función de las excavaciones que realizamos en el interior de la plaza pudimos determinar que el ushnu fue construido sobre una pequeña elevación interna a la plaza, lo que nos lleva a estimar que la superficie superior de la plataforma estuvo elevada al menos $1,5 \mathrm{~m}$ respecto de los niveles de acceso y ocupación en los lados norte y sur. También nos permitió estimar, para los muros perimetrales de la plaza, una altura interior mínima de $2 \mathrm{~m}$.

La plaza presenta un total de nueve accesos. Tres de ellos la vinculan con tres aperturas en el muro perimetral a través de tres senderos. Uno de estos tres accesos (a los que de ahora en adelante llamaremos "públicos") se ubica en el noreste de la plaza y la vincula con la periferia local. El segundo acceso público se encuentra en la sección media del muro sur de la plaza. Un tercer acceso público se ubica en el noroeste de la plaza y está precedido por un pasillo formado por la cara externa de los muros de la kallanka y una de las kanchas. Los seis accesos restantes corresponden a los complejos inkaicos y establecen un vínculo directo con la plaza.

En los últimos años el estudio de las plazas Inka se ha beneficiado con investigaciones respecto de su permeabilidad visual apelando al modelado tridimensional de la arquitectura y la topografía circundante (Acuto y Gifford 2007; Coben 2012; Mackey 2010; Kosiba y Bauer 2013; entre otros). No obstante, la propagación del sonido en asentamientos inkaicos, con la excepción del trabajo de Meddens y Frouin (2011), aún no ha sido investigada. Presentamos a continuación las actividades que realizamos para aproximarnos a las propiedades acústicas de la plaza de Guitián.

\section{Referencias, Materiales y Métodos}

De acuerdo a lo documentado por varios cronistas, las ceremonias, festividades, e incluso las peregrinaciones que se llevaban a cabo bajo imperio involucraban cantos, danzas, y varios instrumentos (principalmente tambores, flautas, y trompetas de varios tamaños y materiales) (ver en Acosta 2008:227 [1590]; Betanzos 1992;I:126127 [1561]; Cieza de León 2005:121, 181 [1553]; Guamán Poma de Ayala 1980;I:228-234 [1615]; Molina 2011:39 [fines del siglo XVI]). Los sonidos no solo eran particularmente relevantes durante las ceremonias, sino que también servían para convocar a las personas a las plazas (Arriaga 1621:8) y cumplían un rol fundamental en acompañar e identificar a las personas en movimientos a gran y pequeña escala (Ávila 1966:145, 147 [1598], Garcilaso de la Vega 1985:6:39, 40 [1609]). Aún hoy, peregrinaciones, homenajes y festividades relativas a deidades locales asociadas a ciertos cerros incluyen danzas y música de flautines y tambores que guían los ritmos de las ceremonias (Bolin 2006:132-133).

Dado que nuestro objetivo principal demanda integrar las vistas y los sonidos potencialmente estimulados por los accesos públicos a la plaza y durante su presencia en ella, realizamos las siguientes actividades (Figura 3).

En primer lugar, tomamos como base del estudio el plano original de Guitián realizado en la década de 1990 por el Proyecto Arqueológico Calchaquí y, junto con datos de excavaciones previas, reestablecimos las coordenadas de la plaza, del ushnu, y el ancho de los accesos con una estación total a fin de afinar la planta previamente realizada. En segundo lugar, también durante los trabajos de campo, testeamos la dispersión del sonido a partir del centro del ushnu. Para ello, trazamos doce ejes desde los vértices del ushnu hasta los muros de la plaza. Sobre cada eje, a intervalos de 3 metros, utilizamos un decibelímetro montado a un trípode para registrar los niveles de presión sonora generados por tres elementos: una trompeta de caracol, un tambor, y la voz de uno de nosotros ${ }^{2}$. Cada eje contó dos puntos de control iniciales adjuntos a la fuente emisora $(0,3 \mathrm{~m}$ y 1 m desde el centro del ushnu) y se completaron los ejes con un punto de medición contra los muros mejor conservados para evaluar la reflexión de las ondas incidentes. Obtuvimos así 171 valores de control para los niveles de presión sonora en diferentes puntos de la plaza. Esto nos permitió generar mapas de isobeles ${ }^{3}$ para la superficie interna de la plaza. Sin embargo, la generación de mapas de isobeles para contextos arqueológicos resulta de utilidad cuando se conservan todas aquellas superficies sobre las cuales se reflejan las ondas sonoras o cuando se realizan mediciones en campos directos. Es decir, sin obstáculos (ver p.ej. Meddens y Frouin 2011). Ante esta doble 


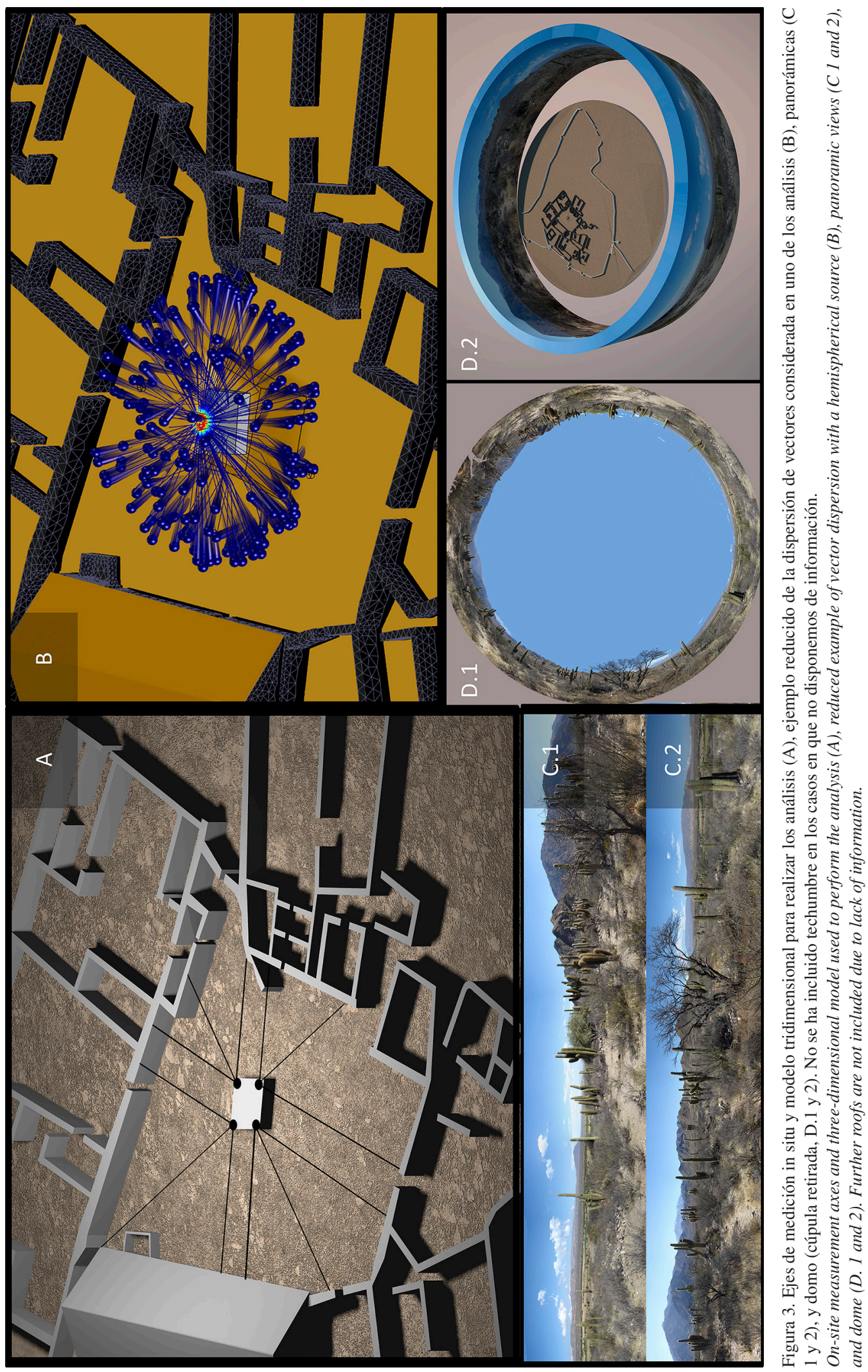


imposibilidad, dada la mala conservación de los muros del asentamiento y la abundante vegetación en ciertas secciones de la plaza, debimos construir un modelo a escala sobre el cual testear la fiabilidad de los valores generados in situ y proyectar el comportamiento de los vectores más allá del campo abierto e inmediato de la plaza. Es decir, hacia las estructuras que la flanquean y hacia el exterior de la plaza incluyendo los conjuntos locales. Para ello realizamos el modelado tridimensional atendiendo a fichas arquitectónicas, datos planimétricos y excavaciones. A fin de determinar la visibilidad del paisaje circundante desde la plaza, completamos el modelo con un domo georreferenciado que integró series de fotografías panorámicas tomadas desde el centro del ushnu. Estas series fueron complementadas con fotografías tomadas desde diferentes puntos en la plaza a fin de controlar el alineamiento de los rasgos arquitectónicos mejor conservados con rasgos prominentes del paisaje circundante. En tercer lugar, atendiendo a los datos generados en campo abierto, modelamos ${ }^{4}$ la dispersión de vectores acústicos desde el centro ushnu hacia las estructuras circundantes. Con propósitos expositivos, todos los resultados presentados a continuación toman como valor de referencia golpes de tambor como fuente emisora ( $80 \mathrm{~dB}$ a $0,3 \mathrm{~m}$ ), ya que, tal como se desprendió de los valores generados in situ, el golpe de tambor es el que menor variabilidad ofrece dados los materiales y la pericia de los ejecutantes. Esto no implica que se asuma que un oficiante posicionado sobre la plataforma estaba tocando un tambor. Ocurre que otros emisores recurrentemente mencionados (en particular la voz para dirigir las actividades) nos presentaron serios desafíos. Varias mediciones de control realizadas en el mismo lugar con la misma fuente emisora, por agotamiento de la voz y falta de pericia en la trompeta de caracol, ofrecieron valores más dispares de lo que estamos dispuestos a aceptar para este estudio.

\section{Resultados: Dispersión Vectorial}

Como se puede observar en las simulaciones de dispersión vectorial (Figura 4), los accesos públicos a la plaza cumplen efectivamente con el propósito de inhibir y canalizar la dispersión de vectores de sonido. En otro trabajo hemos mostrado que la arquitectura de la plaza de Guitián restringía la proyección de la vista al interior de los complejos inkaicos dada la distribución asimétrica de sus accesos (salvo en el caso de la kallanka) (Acuto et al. 2012). Para aquellos que se adentrasen en la plaza, la altura de las paredes aseguraba que la atención se concentrase necesariamente en la plataforma ceremonial, quedando la accesibilidad visual a ciertos rasgos del paisaje circundante reservada al/los oficiantes posicionados sobre el ushnu. La arquitectura de la plaza de Guitián no solo censuraba la posibilidad de proyectar la vista al exterior, sino que aseguraba que el grueso de las reflexiones de los sonidos emitidos se concentrara en su interior (efecto más evidente si se consideraran techumbres adicionales a la kallanka). Claramente, el acceso noreste bloquea la dispersión de vectores hacia la periferia local. El acceso sur habilita una mayor dispersión de vectores hacia afuera de la plaza, pero estos quedan mayoritariamente circunscritos a la inmediatez del acceso. El acceso noroeste no solo "captura" una mayor cantidad de vectores sino que además los canaliza hacia el exterior de la plaza. El modelado de los niveles de presión sonora acumulada en las superficies a partir de la sumatoria de la totalidad de ondas incidentes y reflejadas en todas las superficies refuerza lo expuesto respecto de la capacidad de la plaza para concentrar los sonidos y la capacidad del pasillo noroeste para canalizarlos (Figura 5). No obstante, en este caso, ni la dispersión vectorial ni los niveles de presión ofrecen indicadores suficientes para estimar lo que una persona puede haber oído en su aproximación y permanencia en la plaza. Sí para identificar la incidencia de ciertos rasgos arquitectónicos en la dispersión sonora general.

Entonces, ¿cómo influían las propiedades arquitectónicas de la plaza en el volumen percibido por quienes participasen de las ceremonias auspiciadas allí? ¿Cómo se correlacionan el volumen percibido y las vistas ofrecidas por los accesos públicos a la plaza?

Con el fin de responder a estas preguntas insertamos al modelo 36 sondas cilíndricas no reflectantes; 27 en el interior de la plaza, tres a un metro antes de entrar a la plaza a través de sus accesos públicos, dos adicionales en el pasillo noroeste, una adicional en el camino que precede a la entrada sur, y tres en la periferia local (Figura 6). Las sondas comprenden tres instancias; una de participación pasiva (periferia local), una de aproximación (un metro antes de los accesos), y una de permanencia en la plaza. Cada sonda emula la posición de la cabeza de una persona erguida y movimientos leves 


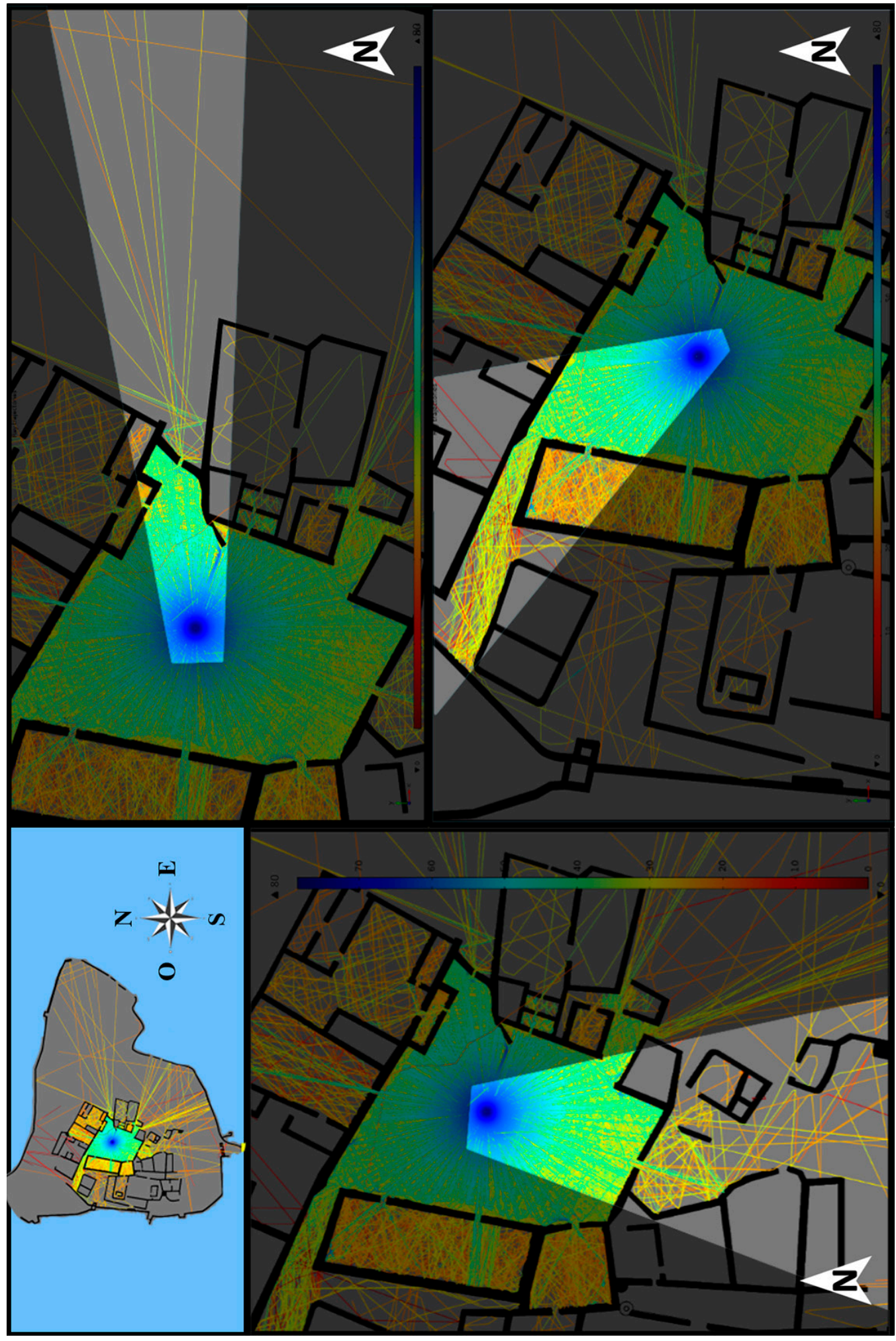

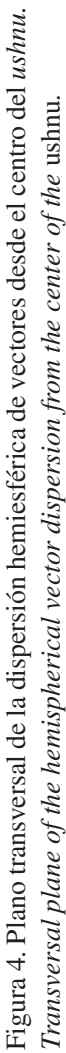




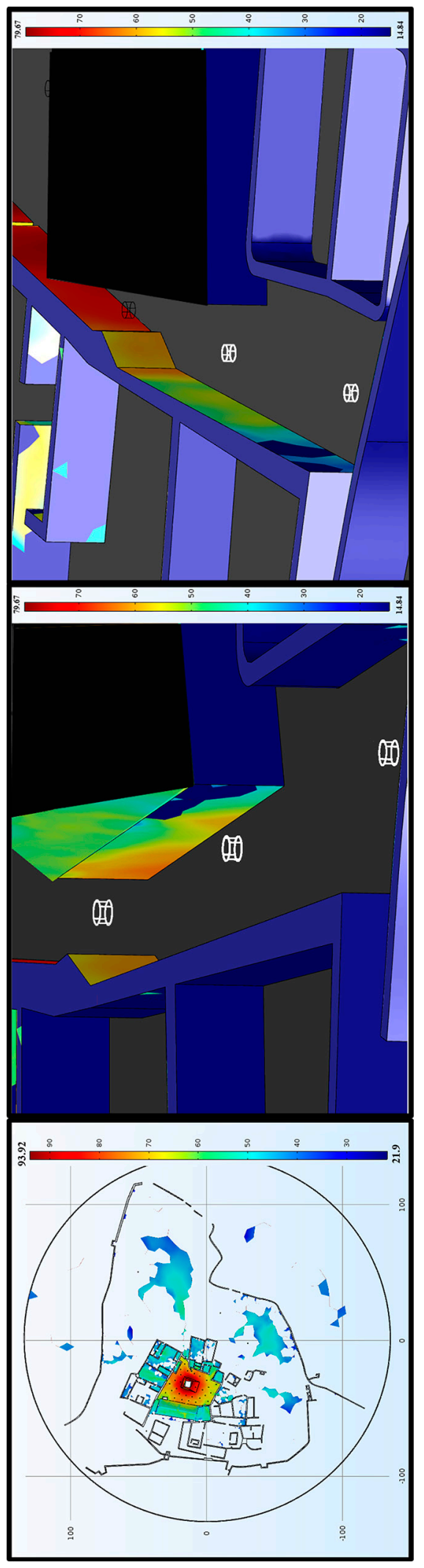

hacia los lados; su centro se ubica a $1,5 \mathrm{~m}$ respecto de la superficie y su volumen comprende un radio horizontal y un eje vertical de $0,3 \mathrm{~m}$.

\section{Dispersión Sonora y Volumen Percibido}

Los resultados (Figura 6) indican que una persona en el interior de la plaza debió haber recibido un nivel de presión sonora medio de 60,98 $\mathrm{dB}$. Es decir, 10,53 dB más que lo que podrían haber recibido aquellos posicionados $1 \mathrm{~m}$ antes de trascender los accesos públicos y 22,16 dB más que aquellos en la periferia local. De acuerdo a estándares psicoacústicos (ver, p.ej. Scharf 1978), los participantes de las ceremonias auspiciadas en la plaza habrían percibido los sonidos con el cuádruple de volumen respecto de aquellos en la periferia local. La arquitectura de Guitián entonces, al circunscribir la accesibilidad visual a su interior e inhibir la dispersión sonora hacia el exterior, limitaba severamente la participación directa e indirecta de esta periferia en las actividades llevadas dentro de la plaza. No obstante, este descenso para la periferia local no implicaba inaudibilidad. Sí reservaba la inmersión auditiva en toda su dimensión a aquellos habilitados para participar de las actividades auspiciadas dentro de la plaza.

Este juego de percepciones visuales y acústicas dispares no se limitaba a crear diferentes experiencias para los habitantes de la periferia local respecto de los participantes de las ceremonias. Por el contrario, se trató de un recurso que también podría haber sido explotado en la aproximación a cada uno de los accesos públicos (Figura 7).

Los accesos públicos a la plaza ofrecían diferentes vistas para quienes se aproximaran a ellos y la posición y ancho del acceso sur, al enmarcar el ushnu, era el único capaz de anticipar la escena y anunciar la importancia de la plataforma para quienes se aproximasen a la plaza. Tanto el acceso noroeste como el acceso noreste inhibían la proyección de la vista hacia el interior de la plaza y no permitían ver la totalidad del ushnu antes de adentrarse en ella. No obstante, afectaban la dispersión sonora de modo muy distinto. De acuerdo a estándares psicoacústicos, una persona en el pasillo noroeste un metro antes de trascender el acceso (sonda $28=$ $55,82 \mathrm{~dB}$ ) y entrar en la plaza podría haber percibido sonidos con más del doble de volumen respecto de una persona tras el acceso noreste (sonda $29=$ $43,79 \mathrm{~dB}$ ). Incluso perceptiblemente superior al 


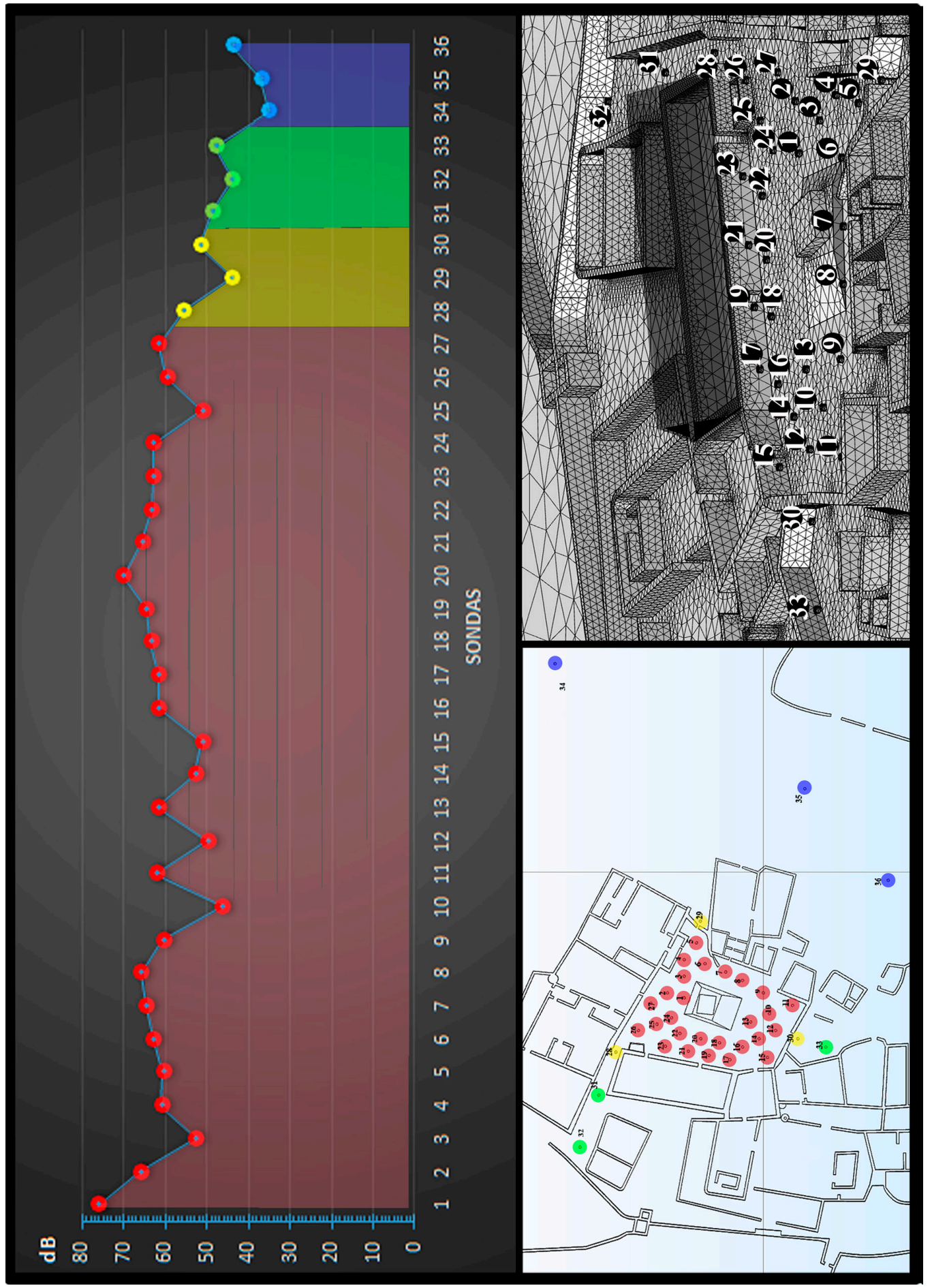

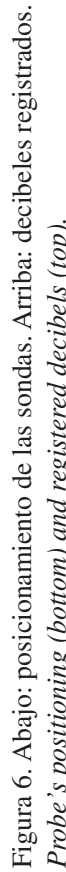



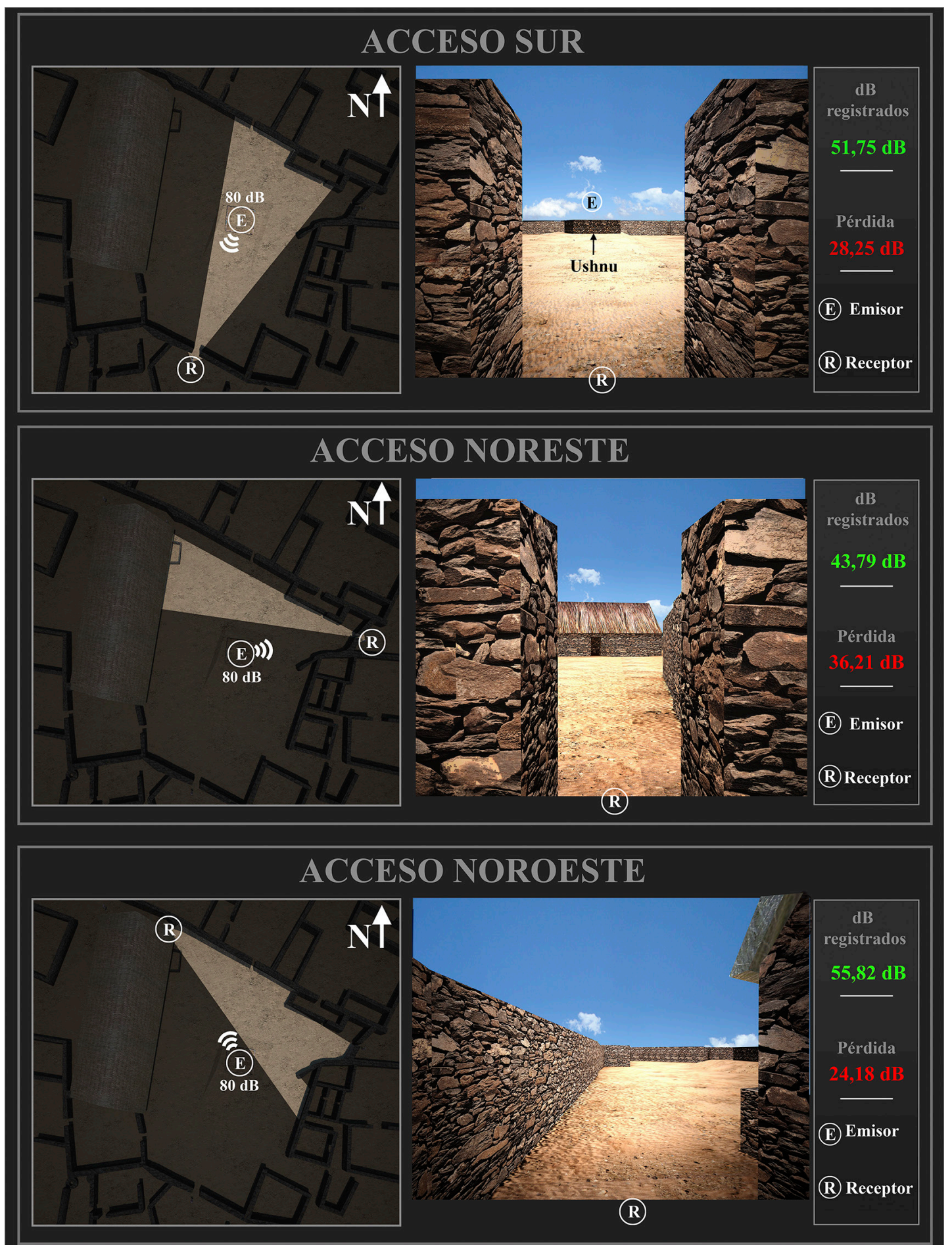

Figura 7. Campo visual habilitado por la arquitectura durante la aproximación a los accesos públicos a la plaza y niveles de presión sonora registrados por las sondas 5 .

Views enabled by architecture while approaching the public accesses to enter the plaza and sound pressure levels registered by the probes. 
volumen percibido antes del acceso sur (sonda 30 $=51,75 \mathrm{~dB}$ ).

Los accesos públicos a Guitián ofrecían entonces tres combinaciones de percepciones visuales y acústicas. Mientras que el evento de ingreso desde el acceso sur habilitaba la participación conjunta de la vista y la audición sin restricciones mayores y el acceso noreste las inhibía, el acceso noroeste combinaba la inhibición de la accesibilidad visual con un incremento relativo en la accesibilidad auditiva.

Así como consideramos el potencial del diseño de la plaza para crear experiencias disímiles para quienes se aproximaran a ella, debemos considerar también la posibilidad de este diseño para anticipar la llegada de las personas. Señalamos que denotar la filiación étnica de los participantes a través de la vestimenta, adornos corporales, danzas, cantos, e instrumentos tradicionales era un aspecto central de estas ceremonias. ¿Cuáles son entonces, si es que existen, las cualidades anticipatorias de los accesos públicos? Para evaluar este escenario hipotético alternativo, invertimos las relaciones entre emisor y receptor. Específicamente, para el caso del pasillo noroeste y el acceso sur (Figura 8).

Los resultados indican que invertir la relación emisor-receptor en el caso del acceso sur implica una diferencia imperceptible de 0,49 dB. En el caso del acceso noroeste, posicionar el emisor dentro del pasillo y un metro antes de trascender el acceso implica una pérdida relativa de $8,84 \mathrm{~dB}$ para un receptor posicionado sobre el ushnu. Esto es, aquel acceso que capturaba los vectores y los canalizaba efectivamente hacia el exterior de la plaza, los mitigaba hacia el interior, por lo que este fenómeno de canalización y el nivel de presión sonora que conlleva era unidireccional; se revelaba efectivo únicamente hacia afuera de la plaza.

\section{Discusión y Consideraciones Finales}

Una de las mayores dificultades a la hora de evaluar espacialidades radica en la incertidumbre asociada al potencial movimiento de las personas, su ubicación y cantidad. Esto es particularmente relevante en el caso de aquellos espacios que tienen la capacidad para albergar una gran cantidad de personas y presentan más de un acceso. A menudo, ciertos rasgos arquitectónicos nos permiten realizar cómodamente asunciones respecto de la posición de los cuerpos y el movimiento en o en torno a ellos (por ejemplo, estructuras y complejos arquitectónicos con un único acceso, sillería, y plataformas con escalinatas). Otras características, como la existencia de accesos múltiples, ofrecen un panorama más complejo. En el caso concreto analizado aquí, podríamos preguntarnos si la accesibilidad física a la plaza estaba pensada para tres grupos diferentes que entraban por los mismos accesos públicos por los que luego abandonaban la plaza, o si acaso fueron pensados como parte de un circuito en donde un grupo entraba por un acceso público y salía por otro.

En el primer escenario hipotético, y de acuerdo a los resultados presentados aquí, la instancia de aproximación a cada acceso creaba condiciones de participación desigual proveyendo estímulos dispares y mostrando claras relaciones de inhibición visual y acústica (noreste), de habilitación (sur), y relaciones intermedias en donde existe una clara intención de inhibir la visualización de la plaza y no la permeabilidad acústica relativa (acceso noroeste). Más allá de estas condiciones desiguales durante la aproximación a la plaza, trascender los tres accesos debió haber supuesto la revelación repentina de la totalidad de la escenografía ceremonial. Señalamos que caminar flanqueado por muros no era habitual en los poblados conglomerados locales de la región. En ellos, a menudo, vías de circulación elevadas recorrían los asentamientos conectando puntos distantes en su interior, circunvalando conjuntos de estructuras, y habilitando la vista de los complejos arquitectónicos lindantes y el interior de las estructuras que no estuviesen techadas. Adicionalmente, muchos de los muros que delimitaban las estructuras locales funcionaban a la vez como senderos auxiliares que asistían a la circulación al interior de los conjuntos arquitectónicos. Aun considerando que toda performatividad en la plaza de Guitián comenzara exclusivamente una vez que todos los visitantes y oficiantes ya estuviesen posicionados, entrar y permanecer en este espacio finamente coreografiado debe haber supuesto un enorme contraste con el habitar local. El posicionamiento y las características de los accesos públicos de Guitián probablemente hayan obedecido a la intención de controlar la inserción de los visitantes locales y el protagonismo de los representantes imperiales. La intervisibilidad entre el ushnu y el acceso sur junto con la capacidad de canalizar sonidos del pasillo noroeste, podría haber asistido a la efectividad de señales visuales y acústicas para "entrar en escena". 


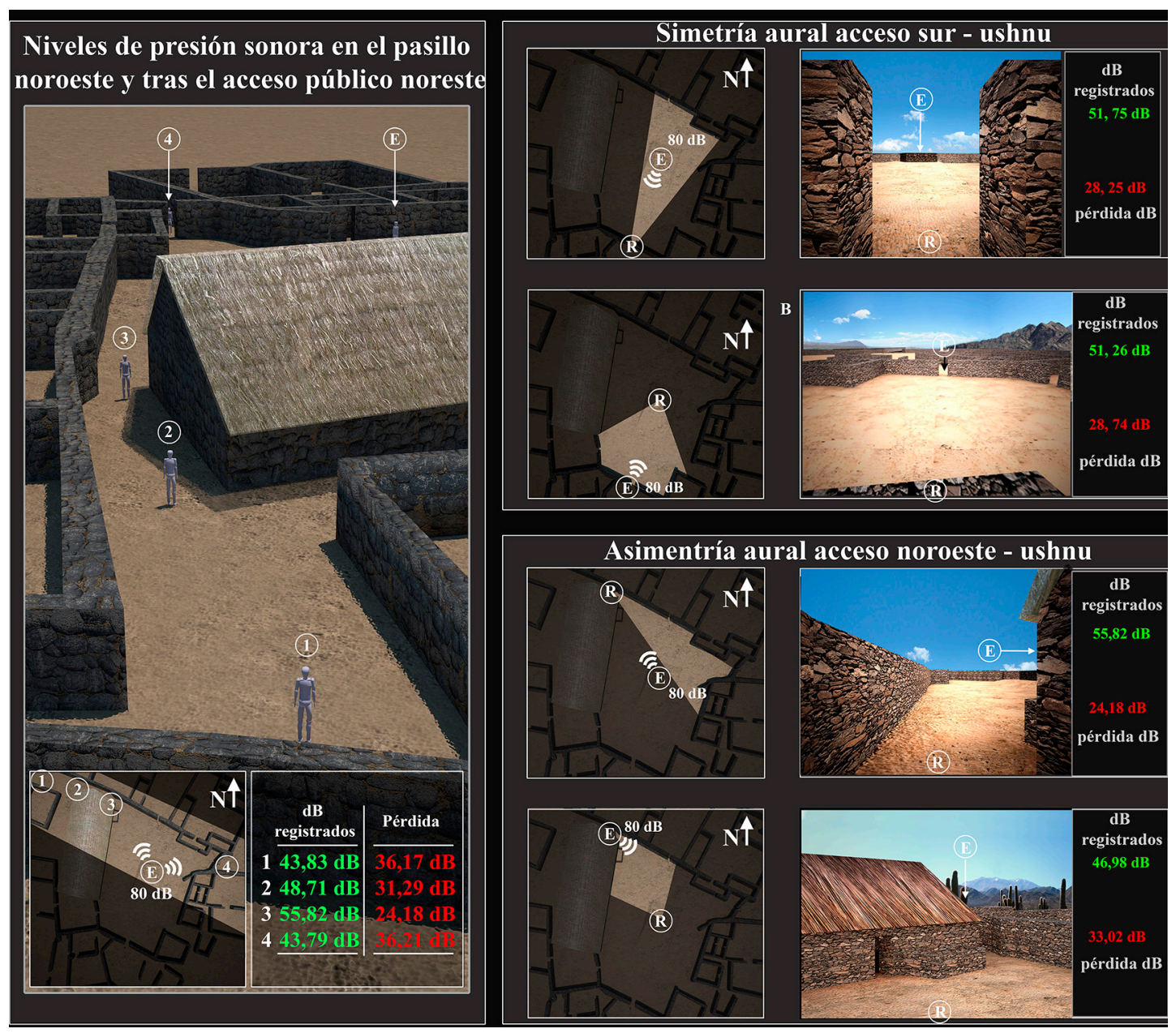

Figura 8. Niveles de presión sonora en las sondas a lo largo del pasillo noroeste (izquierda) y simetrías y asimetrías auditivas dada la inversión de emisores (E) y receptores (R) (derecha).

Sound pressure levels among probes positioned in northwestern hallway (left) and aural symmetries and asymmetries due to inverted emitters $(E)$ and receivers $(R)$ (right).

Crónicas e investigaciones etnográficas documentan que las personas acompañaban las peregrinaciones y participaban en eventos ceremoniales con música de flautines, tambores, y trompetas de caracol mientras se dirigían a ellos. Los resultados presentados aquí indican que, si existía una intención de anticipar la entrada de los participantes, quedaba relegada a la intervisibilidad e interaudibilidad entre quienes se aproximaban al acceso sur y la plataforma ceremonial.

Ciertas características de los accesos a la plaza de Guitián nos llevan a pensar que su diseño podría obedecer a la intención de crear un circuito para sus visitantes. El acceso público noreste bloqueaba la visibilidad del ushnu, restringía severamente la visibilidad del interior de la plaza en el día a día de los sectores locales, y con seguridad no permitía la entrada de más de una persona a la vez. Esto nos sugiere que no fue diseñado para promover una participación activa de los habitantes locales de Guitián quienes, quizás, estaban relegados a asistir esporádicamente a los representantes imperiales. La cualidad de entrada del acceso sur, por su capacidad de enmarcar al ushnu y no romper la intervisibilidad entre él y quienes se aproximaran al acceso, parece difícil de cuestionar. El acceso noroeste se destaca entre los accesos públicos por su capacidad de canalizar los sonidos efectivamente hacia el exterior de la plaza antes que hacia su interior. En un segundo escenario hipotético, mientras que el acceso público 
noreste podría haber tenido una función meramente utilitaria limitada a la colaboración efímera de alguno de los habitantes locales asentados en la periferia, la simetría visual y auditiva del acceso sur podría ser el componente inicial de un circuito en que las personas, luego de adentrarse en la plaza y permanecer en ella, la evacuaban por el pasillo adjunto al acceso noroeste. Hacia el final del pasillo y tras virar hacia el norte para dirigirse a la apertura en el muro perimetral de Guitián, se revelaba por vez primera (en este circuito hipotético y para los visitantes locales), una importante wak'a al menos durante tiempos inkaicos; el cerro Meléndez (a $6.020 \mathrm{msm}$ ), cuya cumbre fue intervenida por los Inkas (Vitry 2008) y, a juzgar por ciertas evidencias de superficie detectadas camino a la cumbre (Jacob et al. 2013), formaba parte de un circuito de peregrinaje que incluía la participación parcial de algunos representantes locales. En todo caso, la planificación que exhibe la plaza de Guitián sugiere una intención de controlar exhaustivamente la accesibilidad física, visual y auditiva a este espacio. Creemos que estos resultados muestran el potencial de análisis complementarios de dispersión sonora para identificar gradaciones y matices que expongan todos los recursos utilizados por el Tawantinsuyu para robustecer las narrativas insertas en aquellos paisajes y lugares que asistieron a la comunicación de la cosmovisión imperial.

Agradecimientos: Este trabajo se llevó adelante con el financiamiento del Consejo Nacional de Investigaciones Científicas y Técnicas (CONICET -Argentina), la ANPCyT (PICT 2013-1659), y el apoyo logístico de la comunidad Diaguita-Kallchaki de La Paya. Las observaciones realizadas por los evaluadores de este manuscrito no solo han mejorado lo expuesto, sino que nos ofrecieron ideas muy interesantes para futuras investigaciones.

\section{Referencias Citadas}

Acosta, J. 2008 [1590]. Historia Natural y Moral de las Indias. Editado por F. del Pino Díaz. Cyan, Proyectos y Producciones Editoriales S.A., Madrid.

Acuto, F.A. 2010. Living under the imperial thumb in the Northern Calchaquí Valley (Argentina). En Distant Provinces in the Inka Empire: Toward a Deeper Understanding of Inka Imperialism, editado por M. Malpass y S. Alconini, pp. 108-150. University of Iowa Press, Iowa City.

Acuto, F.A. y C. Gifford 2007. Lugar, arquitectura y narrativas de poder. Relaciones sociales y experiencia en los centros Inkas de los Andes del Sur. Arqueología Suramericana 3(2): 135-161.

Acuto, F.A., A. Troncoso y A. Ferrari 2012. Recognising strategies for conquered territories: a case study from the Inka North Calchaqui Valley. Antiquity 86:1141-1154.

Alcock, S., T. D’Altroy, K. Morrison y C. Sinopoli 2001. Empires. Perspectives from Archaeology and History. Cambridge University Press, Cambridge.

Ambrosetti, J.B. 1907. Exploraciones arqueológicas en la ciudad prehistórica de La Paya (Valle Calchaquí, Pcia. de Salta). Revista de la Universidad de Buenos Aires VIII:5-534.

Arriaga, P.I. 1621. Extirpación de la Idolatría del Piru. Geronymo de Contreras impressor de libros, Lima.

Ávila, F. 1966 [1598]. Dioses y Hombres de Huarochirí. Traducido por J.M. Arguedas y estudio bibliográfico de P. Duviols. Instituto de Estudios Peruanos, Lima.

Bauer, B. 1992. The Development of the Inca State. University of Texas Press, Austin.

Bell, C. 1992. Ritual Theory, Ritual Practice. Oxford University Press, Oxford.
Betanzos, J. 1992 [1561]. Suma y Narración de los Yngas. Fondo Rotatorio Editorial de Culturas Aborígenes de América, Tomo I. Ariol SRL, Cochabamba.

Bolin, I. 2006. Growing up in a Culture of Respect. Child Rearing in Highland Peru. University of Texas Press, Austin.

Bourdieu, P. 2007. El Sentido Práctico. Siglo Veintiuno Editores, Buenos Aires.

Cieza de León, P. 2005 [1553]. Crónica del Perú. El Señorío de los Incas. Fundación Biblioteca Ayacucho, Caracas.

Coben, L. 2006. Other Cuzcos: replicated theaters of Inka power. En Theaters of Power and Community: Archaeology of Performance and Politics, editado por T. Inomata y L. Coben, pp. 223-260. Altamira Press, Maryland.

Coben, L. 2012. Theaters of Power: Inca Imperial Performance. Phd Dissertation, Penn Libraries, Publicly Accessible Penn Dissertations. http://repository.upenn.edu/edissertations/499/

Connerton, P. 1989. How Societies Remember. Cambridge University Press, Cambridge.

Cosgrove, D. 2008. Geography and Vision. Seeing, Imagining and Representing the World. Tauris \& Co Ltd, Nueva York.

D’Altroy, T. 2002. The Incas. Blackwell Publishers, Oxford.

Fahlander, F. y A. Kjellström 2010. Beyond sight: Archaeologies of sensory perception. En Making Sense of Things. Archaeologies of Sensory Perception, editado por F. Fahlander y A. Kjellström, pp. 51-68. Postdoctoral Archaeological Group, University of Stockholm, Stockholm.

Farrington, I. y J. Zapata 2003. Nuevos cánones de arquitectura inka: investigaciones en el sitio de Tambokancha-Tumibamba, Jaquijahuana, Cuzco. Boletín de Arqueología PUCP 7:57-77. 
Garcilaso de la Vega, I. 1985 [1609]. Comentarios Reales de los Incas. Libro sexto. Colección $\mathrm{N}^{\circ} 5$. Fundación Biblioteca Ayacucho, Caracas.

González, A.R. y P.P. Díaz 1992. Notas arqueológicas sobre la "Casa Morada", La Paya, pcia. de Salta. Estudios de Arqueología 5:9-61.

Guamán Poma de Ayala, F. 1980 [1615]. Nueva Corónica y Buen Gobierno. Tomo I. Siglo XXI, México, D.F

Hamilakis, Y. 2002. The past as oral history. Towards an archaeology of the senses. En Thinking through the Body. Archaeologies of Corporeality, editado por Y. Hamilakis, M. Pluciennik y S. Tarlow, pp. 121-136. Kluwer Academic/Plenum Publishers, Londres.

Hamilakis, Y. 2011. Archaeologies of the senses. En The Oxford Handbook of the Archaeology of Ritual and Religion, editado por T. Insoll, pp. 208-225. Oxford University Press, Oxford.

Harvey, D. 1994. The social construction of space and time. A relational theory. Geographical Review of Japan 67(2):126-135.

Howes, D. 2006. Scent, sound and synaesthesia. Intersensoriality and material culture theory. En Handbook of Material Culture, editado por C. Tilley, W. Keane, S. Küchler, M. Rowlands y P. Spyer, pp. 161-172. Sage, Londres.

Hyslop, J. 1990. Inka Settlement Planning. University of Texas Press, Austin.

Ingold, T. 2010. Footprints through the weather-world: walking, breathing, knowing. Journal of the Royal Anthropological Institute 16:121-139.

Inomata, T. y L. Coben (eds.) 2006. Archaeology of Performance: Theaters of Power, Community, and Politics. Altamira Press, California.

Jacob, C., I. Leibowicz, F. Acuto y R. Moyano 2013. Paisaje ritual y marcadores astronómicos en el sitio Uña Tambo, Nevados de Cachi, Salta, Argentina. Arqueología y Sociedad 26:289-300.

Karampatzakis, P. y V. Zafranas 2009. Did Hades accept visitors? The acoustical properties of the underground crypt of the Acheron Nekromanteion, Preveza, Greece. 15th Annual Meeting of the European Association of Archaeologists 15:1-12.

Kosiba, S. y A. Bauer 2013. Mapping the political landscape: Toward a GIS analysis of environmental and social difference. Journal of Archaeological Method and Theory 20:61-101.

Leibowicz, I. 2012. Ideología y espacio: conquista inca en la quebrada de Humahuaca, Jujuy, Argentina. Revista Chilena de Antropología 25:65-91.

Mackey, C. 2010. The socioeconomic and ideological transformation of Farfán under Inka rule. En Distant Provinces in the Inka Empire. Toward a deeper Understanding of Inka Imperialism, editado por M. Malpass y S. Alconini, pp. 221-259. University of Iowa Press, Iowa City.

Makowski, K., M.F. Córdova, P. Habetler y M. Lizárraga 2005. La plaza y la fiesta: reflexiones acerca de la función de los patios en la arquitectura pública prehispánica de los períodos tardíos. Boletín de Arqueología PUCP 9:297-333.

McBride, A. 2014. The acoustics of archaeological architecture in the Near Eastern Neolithic. World Archaeology 46:349-361.
Meddens, F.M., N.P. Branch, C. Vivianco Pomacanchari, N. Riddiford y R. Kemp 2008. High altitude ushnu platforms in the department of Ayacucho Perú, structure, ancestors and animating essence. En Pre-Columbian Landscapes of Creation and Origin, editado por J.E. Staller, pp. 315-355. Springer, New York.

Meddens, F. y M. Frouin 2011. Inca sacred space, platforms and their potential soundscape. Preliminary observations at usnu from Ayacucho. Revista Haucaypata, Investigaciones Arqueológicas del Tahuantinsuyo 1:24-40.

Molina, C. 2011 [fines del S XVI]. Account of the Fables and Rites of the Incas. Traducido y editado por B.S. Bauer, V. Smith-Oka y G.E. Cantarutti. University of Texas Press, Austin.

Monteverde Sotil, L.R. 2011. La configuración arquitectónica de los ushnus como espacios de libaciones y ofrendas líquidas durante el Tahuantinsuyo. Bulletin de l'Institut Français d'Études Andines 40:31-80.

Moore, J. 1996. Architecture and Power in the Ancient Andes. The Archaeology of Public Buildings. Cambridge University Press, Cambridge.

Morris, C. y A. Covey 2003. La plaza central de Huánuco Pampa: Espacio y transformación. Boletín de Arqueología PUCP 7:133-149.

Morris, C., A. Covey y P. Stein 2011. The Huánuco Pampa Archaeological Project Volume I: The Plaza and Palace Complex. American Museum of Natural History, Nueva York.

Morris, I. y W. Scheidel (eds.) 2009. The Dynamics of Ancient Empires: State Power from Assyria to Byzantium. Oxford University Press, Nueva York.

Murra, J. 1978. La Organización Económica del Estado Inca. Siglo Veintiuno, México, D.F.

Nielsen, A.E. 2006. Plazas para los antepasados: Descentralización y poder corporativo en las formaciones políticas preinkaicas de los Andes circumpuneños. Estudios Atacameños 31:63-89.

Niles, S. 1999. The Shape of Inca History. Narrative and Architecture in an Andean Empire. University of Iowa Press, Iowa City.

Pallasmaa, J. 2005. The Eyes of the Skin. Architecture and the Senses. Wiley-Academy, Chichester.

Pijanowski, B., L. Villanueva-Rivera, S. Dumyahn, A. Farina, B. Krause, B. Napoletano, S. Gage y N. Pieretti 2011. Soundscape ecology: The science of sound in the landscape. BioScience 61(3):203-216.

Pino Matos, J. 2005. El ushnu y la organización espacial astronómica en la sierra central del Chinchaysuyu. Estudios Atacameños 29:143-161.

Reznikoff, I. 2012. On the sound related to painted caves and rocks. Sounds like Theory. XII Nordic Theoretical Archaeology Group Meeting in Oulu 25. Monographs of the Archaeological Society of Finland 2, editado por J. Ikäheimo, A. Salmi y T. Äikäs, pp. 101-109. The Archaeological Society of Finland, Helsinki.

Schafer, R.M. 1967. Ear Cleaning. Notes for an Experimental Music Course. Clark \& Cruickshank, a Division of Berandol Music Limited, Toronto. 
Scharf, B. 1978. Loudness. En Handbook of Perception, editado por E.C. Carterette y M.P. Friedman, Volumen 4, pp. 187-234. Academic Press, Nueva York.

Soja, E. 1989. Postmodern Geographies: The Reassertion of Space in Critical Social Theory. Verso, London.

Southworth, M. 1969. The sonic environment of cities. Environment and Behavior 1:49-70.

Staller, J.E. 2008. Dimensions of place: The significance of centers to the development of Andean civilization: An exploration of the ushnu concept. En Pre-Columbian Landscapes of Creation and Origin, editado por J. E. Staller, pp. 269-313. Springer, New York.

Stanish, C. 1992. Ancient Andean Political Economy. University of Texas Press, Austin.
Tarlow, S. 2000. Emotion in archaeology. Current Anthropology 41:713-746.

Truax, B. 1984 [1978]. Acoustic Communication. Ablex Publishing Corporation, New Jersey.

Vitry, C. 2008. El Nevado de Cachi. Palloni Ediciones, Río Cuarto, Córdoba.

Watson, A. y D. Keating 1999. Architecture and sound: an acoustic analysis of megalithic monuments in prehistoric Britain. Antiquity 73:325-336.

Williams, V. 2004. Poder estatal y cultura material en el Kollasuyu. Boletín de Arqueología PUCP 8:209-246.

Zuidema, T. 1989. El ushnu. En Reyes y Guerreros: Ensayos de Cultura Andina, compilado por M. Burga, pp. 402-454. Fomciencias, Lima.

\section{Notas}

1 Las excavaciones realizadas en uno de estos complejos indican que se trata de estructuras que combinan la construcción de muros dobles con el excavado y revestido de superficies con rodados fluviales localmente disponibles y sin modificar. Las plantas son subrectangulares, subcuadrangulares y circulares, con pocas divisiones internas cuando presentes, $\mathrm{y}$ circunvaladas por senderos elevados respecto de su superficie interna. Debemos mencionar también que estos complejos presentan unas pocas estructuras cuya arquitectura sugiere que se trata de adiciones inkaicas.

2 Los tres elementos son recurrentemente mencionados en las crónicas, por lo que nos pareció idóneo utilizarlos como punto de partida para la generación de datos in situ así como para el posterior modelado de la dispersión en un intervalo espacial más amplio. Dadas las condiciones climáticas de la zona, estas mediciones se realizaron por la mañana a fin de mitigar la dispersión sonora generada por fuertes vientos encajonados provenientes del oeste-noroeste. La temperatura ambiente registrada fue de $20^{\circ} \mathrm{C}$ y el sonido ambiente registrado fue de $30,7 \mathrm{~dB}$.

3 Un mapa de isobeles consiste a menudo en la expresión bidimensional de la variación de los niveles de presión sonora a partir de la conexión de aquellos puntos en los que es equivalente.

4 A este efecto se testearon diversos programas de modelado y representación (DISIA, I-SIMPA, CATT-Acoustic, COMSOL, ODEON, entre otros). Estos programas habilitan la definición de variables fundamentales a considerar, como ser: presión atmosférica, temperatura, humedad relativa, coeficientes de absorción de los materiales de construcción, velocidad de propagación del sonido en los materiales, y probabilidad de reflexión especular y difusa de acuerdo a la heterogeneidad de las superficies de reflexión, además de fenómenos de reflexión especular, difusa, difracción y refracción.

5 Las texturas utilizadas en el renderizado son a modo ilustrativo. 
